\title{
STRONG BERTINI THEOREMS
}

\author{
STEVEN DIAZ AND DAVID HARBATER
}

\begin{abstract}
We show that the singular locus of the general member of a linear system has dimension less than that predicted by Bertini's theorem, provided that the base locus is scheme-theoretically smooth. As corollaries, we obtain a result about complete intersection varieties containing a given subvariety and a result concerning liaison.
\end{abstract}

\section{INTRODUCTION}

The classical Bertini theorem in characteristic zero states that the general element of a linear system of divisors on a variety $X$ is nonsingular away from the base locus of the linear system and the singularities of $X$. In characteristic $p$, if the linear system is assumed to be very ample, then the same conclusion is obtained. In this paper we show that if the base locus of the linear system is scheme-theoretically reduced and nonsingular, the singularities of the general element of the linear system have dimension less than that predicted by Bertini, and in fact (when such singularities actually occur) this dimension may be computed exactly. We present two applications of these strengthened Bertini theorems: In $\S 3$ we generalize a theorem of Nishi [N] about finding nonsingular complete intersection varieties that contain a given nonsingular variety. In $\S 4$ we obtain some results about when a given nonsingular subvariety of projective space can be directly linked in liaison to another nonsingular variety.

We fix our conventions for the paper: The field $K$ will be algebraically closed and of arbitrary characteristic (although we will sometimes explicitly add the assumption that char $K=0$ and will also sometimes consider nonalgebraically closed subfields of $K$ ). A variety $X$ over $K$ will be an integral, separated scheme of finite type over $K$. The singular points of $X$ are the points whose local rings are not regular, and $X$ is nonsingular if all its local rings are regular. The dimension of a scheme is the maximum of the dimensions of its irreducible components. When we say that the "general element" of a linear system has a certain property, we mean that there is a Zariski dense open subset $U$ of the projective space parametrizing the linear system such that all divisors corresponding to points in $U$ have the given property.

Received by the editors February 17, 1989.

1980 Mathematics Subject Classification (1985 Revision). Primary 14C20; Secondary 14B05.

Key words and phrases. Bertini theorem, complete intersection, liaison.

The second author was partially supported by National Science Foundation Grant DMS8514835. 
The authors would like to thank Donu Arapura, Dale Cutkosky, Ron Donagi, and Joe Harris for helpful discussions during the investigations that led to this paper.

\section{StRONG BERTINI THEOREMS}

In this section we show the main result of the paper (Theorem 2.1), concerning the singularities of the general member of a linear system whose base locus is reduced and nonsingular. In the characteristic 0 case, we show that the codimension of this singular locus in each component of the base locus is equal to the codimension of that component of the base locus in the ambient variety. In many cases this implies that the general member is nonsingular; see Corollary 2.4 .

For this section we fix a linear system $\Lambda$ of effective Cartier divisors on a complete variety $X$. Let $B$ be the scheme-theoretic base locus of $\Lambda, \pi: X^{\prime} \rightarrow$ $X$ the blow-up of $X$ along $B$, and $E$ the exceptional divisor of this blow-up. Let $X^{0}$ be the set of nonsingular points of $X$. Let $D$ be a general element of $\Lambda$, and $D_{s}$ the set of singular points of $D$. Let $B^{0}$ be an irreducible component of $B \cap X^{0}$. It might be that $B^{0} \cap D_{s}$ is empty; let us assume that this is not the case and let $S$ be an irreducible component of $S^{0}=B^{0} \cap D_{s}$. Let $a$ be the codimension of $B^{0}$ in $X$ and let $b$ be the codimension of $S$ in $B^{0}$.

(2.1) Theorem. In the preceding situation, assume either that $K$ has characteristic zero or that the linear system $\pi^{*} \Lambda-E$ is very ample on $X^{\prime}$. Then

(1) $D_{s} \cap X^{0} \subset B \cap X^{0}$.

(2) If we further assume that $B \cap X^{0}$ is reduced and nonsingular, then $a=b$.

Proof. Because $B$ is the scheme-theoretic base locus of $\Lambda$, the linear system $\pi^{*} \Lambda-E$ is base point free. Also, when the characteristic of $K$ is not zero we have assumed that $\pi^{*} \Lambda-E$ is very ample. Thus, in any characteristic the classical Bertini theorem applies to tell us that a general element $D^{\prime}$ of the linear system $\pi^{*} \Lambda-E$ is nonsingular away from the singularities of $X^{\prime}$. All singularities of $X^{\prime}$ and thus $D^{\prime}$ lie over $B$ or singularities of $X$. We may then take our general divisor $D$ of $\Lambda$ to be $\pi\left(D^{\prime}\right)$ and from the previous few sentences conclude that $D$ satisfies (1). To prove (2) note that the additional assumptions on $B$ say that all singularities of $X^{\prime}$ and thus $D^{\prime}$ lie over singularities of $X$. We must determine when a nonsingular point of $D^{\prime}$ lying over a nonsingular point of $X$ contained in $B$ maps to a singular point of $D$.

(2.2) Claim. In case (2) of (2.1) let $p$ be a point of $B \cap X^{0}$. Then $p$ is a singular point of $D$ if and only if $D^{\prime}$ contains the entire fiber $\pi^{-1}(p)$.

Proof of claim. This is an explicit local calculation and may be done in the complete local ring of $X$ at $p$. Let $z_{1}, \ldots, z_{n}$ be a system of local coordinates on $X$ at $p$. We may assume $B$ is given by $z_{k+1}=\cdots=z_{n}=0$ and $D$ by $f\left(z_{1}, \ldots, z_{n}\right)=0$. Because $B \subset D$, each monomial of $f$ will be divisible by 
some $z_{i}$ with $i \geq k+1$. If all divisors in $\Lambda$ were singular everywhere on $B$, then $B$ could not be reduced; thus we may assume that $D$ is nonsingular on an open dense subset of $B$. By taking partial derivatives of $f$ we may see that this implies that at least one monomial of $f$ has degree one in $z_{k+1}, \ldots, z_{n}$. Note that $D$ is singular at $p=(0, \ldots, 0)$ if and only if all monomials of $f$ have degree at least two.

Now blow up $B$. To determine the equation of $D^{\prime}$ on a typical affine patch $U_{j}$ of the blow-up, we make the following substitutions into $f$ :

$$
\begin{aligned}
& z_{i} \mapsto z_{i} ; \quad i=1, \ldots, k ; \\
& z_{i} \mapsto z_{i} z_{j} ; \quad i=k+1, \ldots, \hat{j}, \ldots, n ; \\
& z_{j} \mapsto z_{j} .
\end{aligned}
$$

The equation for $E$ is $z_{j}=0$. From what we have said about $f$, we see that after making the preceding substitution into $f$ it will be divisible by $z_{j}$ exactly once. Call the quotient $\bar{f}$; this is the equation for $D^{\prime}$. The equations for $\pi^{-1}(p)$ are $z_{1}=\cdots=z_{k}=z_{j}=0$. Thus $D^{\prime}$ will fail to contain $\pi^{-1}(p)$ if and only if $\bar{f}$ contains a constant term or a monomial containing only $z_{i}$ 's for $i \geq k+1, i \neq j$. Reconsidering how monomials of $\bar{f}$ arise from monomials of $f$, we see that this is the case if and only if $f$ has a monomial of degree exactly one, that is, if and only if $D$ is nonsingular at $p$. This completes the proof of the claim.

To see how often $D^{\prime}$ can contain a fiber of $\pi$ over a point of $B$ we consider the morphism $\phi: X^{\prime} \rightarrow \mathbf{P}^{N}$ given by the linear system $\pi^{*} \Lambda-E$. Because of the local nature of what we are trying to show, we may assume that $X$ is nonsingular and $B$ has only one component. If $N=a-1$ then $B$ is a complete intersection of $a$ elements of $\Lambda$ and all divisors of $\Lambda$ must be nonsingular everywhere on $B$; so we may assume $N>a-1$. For each point $p$ of $B, \phi$ embeds $\pi^{-1}(p)$ in $\mathbf{P}^{N}$ as an $a-1$ plane. This follows from standard knowledge about blowups: $\pi^{-1}(p)$ is the projectivization of the normal space to $B$ in $X$ at $p$, the divisors of $\Lambda$ generate the ideal of $B$ in $X$ at $p$, and $\pi^{*} \Lambda-E$ restricts to the bundle $\mathscr{O}(1)$ on $\pi^{-1}(p)=\mathbf{P}^{a-1}$.

Let $\mathbf{P}^{N^{*}}$ be the projective space of hyperplanes in $\mathbf{P}^{N}$. Consider the product $B \times \mathbf{P}^{N^{*}}$ with projections $\pi_{1}$ and $\pi_{2}$ onto the first and second factors. Inside $B \times \mathbf{P}^{N^{*}}$ define $I=\left\{(b, H): \pi^{-1}(b) \subset H\right\}$. For any point $b$ of $B, \pi_{1}^{-1}(b)=$ hyperplanes containing a fixed $a-1$ plane, so dimension $\pi^{-1}(b)=N-a$. This says that $\operatorname{dim} I=\operatorname{dim} B+N-a$. So the dimension of a general fiber of $\pi_{2}$ is at most $\operatorname{dim} B-a$. But by the claim $(2.2) \pi_{1}\left(\pi_{2}^{-1}(H)\right)$ is the singular locus of the general $D$ corresponding to this general $H$. This shows that $b \geq a$.

Finally, to show that $b \leq a$, let $\mathscr{I}$ be the ideal sheaf of $B$ in $X$ and $\mathscr{L}=\mathscr{O}(D)$, so that $\Lambda$ is contained in the complete linear system corresponding 
to $\mathscr{L}$. Consider the following exact sequence of sheaves on $B$ :

$$
0 \rightarrow \mathscr{I}^{2} \otimes \mathscr{L} \rightarrow \mathscr{I} \otimes \mathscr{L} \rightarrow \mathscr{I} / \mathscr{I}^{2} \otimes \mathscr{L} \rightarrow 0 .
$$

The sheaf $\mathscr{I} / \mathscr{I}^{2}$ is the conormal bundle to $B$ in $X$; therefore, $\mathscr{I} / \mathscr{I}^{2} \otimes \mathscr{L}$ is a vector bundle of rank $a$. A divisor $D$ in $\Lambda$ gives a global section of $\mathscr{I} \otimes \mathscr{L}$ and therefore a global section of $\mathscr{I} / \mathscr{I}^{2} \otimes \mathscr{L}$ vanishing exactly on the singularities of $D$ contained in $B$. The zero locus of a section of a vector bundle of rank $a$ on a nonsingular variety is either empty or has codimension at most $a$. Q.E.D.

Remark. The proof of (2.1) also shows that for any divisor $D \in \Lambda$, if $D_{s} \cap B^{0}$ has codim $>a$ in $B^{0}$ then $D_{s} \cap B^{0}$ is empty.

(2.4) Corollary. Under the hypotheses of Theorem 2.1(2), if the dimension of a component of $B \cap X^{0}$ is less than $\frac{1}{2} \operatorname{dim} X$, then a general element of the linear system is nonsingular along this component.

Warning. Even if the dimension of a component of $B \cap X^{0}$ is greater than $\frac{1}{2} \operatorname{dim} X$ it may be that a general element of $\Lambda$ is nonsingular along this component. (For example, consider a nonsingular complete intersection of nonsingular hypersurfaces in $\mathbf{P}^{n}$.) See (2.5) and (3.4) for more on this.

By looking more carefully at the proof of Theorem 2.1, we can say even more about the singularities of our general $D$-in particular that the singular locus is itself nonsingular. To do this we describe the singularities separately on each irreducible component of $B \cap X^{0}$. Let $B^{0}$ be a fixed irreducible component of $B \cap X^{0}$ of dimension $d$ and codimension $a$ in $X$ and let $S^{0}=B \cap D_{s}$. Denote by $A_{i}\left(B^{0}\right)$ the $i$ th Chow group of $i$-cycles modulo rational equivalence, as defined for instance in [F]. Let $F$ be the twisted conormal bundle $\mathscr{I} / \mathscr{I}^{2} \otimes \mathscr{L}$ of (2.3) for $B^{0}$ in $X$, and let $c_{i}(F)$ be the $i$ th Chern class of $F$. For our general divisor $D$ let $s_{D}$ be a section of $F$ induced by $D$ (so that $s_{D}$ is determined up to a nonzero constant multiple). Let $Z$ be the zero scheme of $s_{D}$ and let $[Z]$ be the class of the cycle associated to $Z$ in $A_{d-a}\left(B^{0}\right)$ as defined for instance in $[F]$.

(2.5) Corollary. With the preceding notation and under the assumptions of part (2) of (2.1) the following are true:

(a) Set theoretically, $Z=S^{0}$.

(b) $[Z]=c_{a}(F) \cap\left[B^{0}\right]$ in $A_{d-a}\left(B^{0}\right)$.

(c) $S^{0}$ is empty if and only if $c_{a}(F)=0$.

(d) The singularities of $D$ in $X^{0}$ are all double points.

(e) $Z$ is a nonsingular reduced scheme. Thus $[\mathrm{Z}]$ is a sum of disjoint nonsingular varieties each counted with multiplicity one.

Proof. Part (a) is clear from the discussion surrounding (2.3). Parts (b) and (c) follow from standard knowledge about Chern classes [F, Example 3.2.16, 
Appendix A.7] and the fact (2.1(2)) that if nonempty, $Z$ has the expected dimension. Parts (d) and (e) will follow from a closer look at the local calculation used in the proof of (2.2). Part (d) is straightforward: If $p=(0, \ldots, 0)$ were a triple point or worse of $D$, then all the monomials of $f$ would have degree at least 3. This would imply that all monomials of $\bar{f}$ have degree at least 2 , meaning that $D^{\prime}$ is singular, contrary to assumption. Part (e) requires more work:

First, let us write down the section $s_{D}$ in terms of our local coordinates $z_{i}$ and local equation $f$. Keep the notation of the proof of (2.2). Write $f$ as follows: $g_{k+1}\left(z_{1}, \ldots, z_{k}\right) z_{k+1}+\cdots+g_{n}\left(z_{1}, \ldots, z_{k}\right) z_{n}+$ terms of degree at least two in $z_{k+1}, \ldots, z_{n}$. The terms of degree one in $z_{k+1}, \ldots, z_{n}$ give a local expression for $s_{D}$. The zero scheme $Z$ is the subscheme of $B^{0}$ defined by the vanishing of the $g_{i}$ 's. We may assume dimensions are such that $D$ does have singular points. Thus we may assume $k \geq n-k$ and $p=(0, \ldots, 0)$ is a singular point of $D$, so that all the $g_{i}$ 's have constant term zero. To show that $Z$ is reduced and nonsingular we must show that the matrix

$$
J=\left[\frac{\partial g_{i}}{\partial z_{l}}(0, \ldots, 0)\right], \quad k+1 \leq i \leq n, 1 \leq l \leq k,
$$

has rank $n-k$.

The linear system $\pi^{*} \Lambda-E$ on $X^{\prime}$ is either very ample or base point free (depending on our assumptions according to the characteristic of $K$ ). It will restrict to a linear system on $E$ which is again either very ample or base point free. Applying classical Bertini to this restricted linear system, we conclude that, for general $D^{\prime}, D^{\prime} \cap E$ is reduced and nonsingular over $B^{0}$. Now suppose the matrix $J$ of (2.6) does not have rank $n-k$. This means that the system of linear equations

$$
J\left[\begin{array}{c}
z_{k+1} \\
\vdots \\
z_{n}
\end{array}\right]=\left[\begin{array}{c}
0 \\
\vdots \\
0
\end{array}\right]
$$

has at least a one-dimensional family of solutions. In particular, for some $j$ it has a solution of the form $z_{j}=1$ and $z_{i}=x_{i}, i \geq k+1, i \neq j$. One then checks by looking at the appropriate matrix of partial derivatives that on the patch of the blow-up $U_{j}$ where $E=\left\{z_{j}=0\right\}$, the point $z_{1}=\cdots=z_{k}=$ $z_{j}=0, z_{i}=x_{i}, i \geq k+1, i \neq j$, is a singular point of $D^{\prime} \cap E$, contrary to assumption. Q.E.D.

Remarks. (a) A divisor $D$ in $\Lambda$ satisfying the conclusions of (2.1) might not satisfy all the conclusions of (2.5). In the proof of (2.1) we only need that $D^{\prime}$ is nonsingular, whereas in the proof of (2.5) we also need that $D^{\prime} \cap E$ is nonsingular over $B^{0}$. Note also that the singularities of our general $D$ over $B^{0}$ have resolutions with projective spaces as fibers, and thus they are rational double points. 
(b) Concerning possible generalizations of (2.1), note that standard counterexamples show that in characteristic zero all divisors of $\Lambda$ may have moving singularities contained in the base locus. Similarly, in characteristic $p>0$ without a very ampleness assumption, all divisors may have moving singularities outside the possibly empty base locus. See [H1, exercises III.10.7, III.10.8]; $[Z$, p. 140]. Also, it is clear that all divisors of $\Lambda$ will be singular where they meet singularities of $X$. But see Proposition 2.7 which follows.

Keep all of the assumptions of $(2.1)(2)$ except allow $B$ to have singularities outside the singularities of $X$. Allow these singularities to be either singularities of the underlying reduced scheme of $B$ or points at which $B$ is not reduced. The singularities of a general $D$ contained in the locus where both $X$ and $B$ are nonsingular still obey (2.1) and (2.5). As for the singularities of a general $D$ which lie in the singular locus of $B \cap X^{0}$, we have the following result.

(2.7) Proposition. In the preceding situation, let $Y$ be an irreducible component of the singular locus of $B \cap X^{0}$ and let $Y_{1}$ be an irreducible component of $D_{s} \cap Y$ for a general $D$. Assume that for some nonempty Zariski open set $U \subset Y$ and each closed point $y \in U$, the dimension of the tangent space to $B$ at $y$ is strictly less than the dimension of $X$. Then $Y_{1}$ has codimension at least one in $Y$.

Proof. It is sufficient to show that for each $y \in U$ at least one $D$ (possibly dependent on $y$ ) is nonsingular at $y$; this of course implies that a general $D$ is nonsingular at $y$. If every $D$ were singular at $y$, then every $D$ would have tangent space at $y$ equal to the tangent space to $X$ at $y$. This would imply that $B$, which is the intersection of all the $D$ 's, would have tangent space at $y$ equal to the tangent space to $X$ at $y$. This is contrary to our assumption on the dimensions of the tangent spaces to $B$ at points of $U$. Q.E.D.

To end this section we consider Bertini's theorem for nonalgebraically closed fields.

(2.8) Proposition. Let $L$ be any infinite subfield of the algebraically closed field $K$, and assume that the linear system $\Lambda$ is defined over $L$. Then a general divisor $D$ satisfying the conclusions of (2.1), (2.5), and (2.7) may be taken to be defined over $L$.

Proof. By assumption, the linear system $\Lambda$ over $K$ is induced by a linear system $\Delta$ over $L$. Here $\Delta$ may be identified with a projective space $\mathbf{P}_{L}^{n}$, and the induced system $\Lambda$ over $K$ may compatibly be identified with $\mathbf{P}_{K}^{n}=$ $\mathbf{P}_{L}^{n} \times{ }_{L} K$. Note that after replacing $K$ by the algebraic closure of $L$ in $K$, we may assume that $K$ is algebraic over $L$.

Now according to $(2.1),(2.5)$, and (2.7), there is a nonempty Zariski open set $U$ in $\mathbf{P}_{K}^{n}$, in which any $K$-valued point corresponds to a divisor $D$ having the desired properties. After shrinking $U$, we may assume that $U$ is a basic open set, whose complement is the locus of a homogeneous polynomial $F \in K\left[X_{0}, \ldots, X_{n}\right]$. Since $F$ has only finitely many coefficients, 
$F \in M\left[X_{0}, \ldots, X_{n}\right]$ for some subfield $M \subset K$ which is finite over $L$. After enlarging $M$, we may assume that the field extension $L \subset M$ is normal. Let $U_{M}$ be the complement of the zero locus of $F$ in $\mathbf{P}_{M}^{n}$. Then $U_{M}$ is open, and $U_{M} \times_{M} K=U$.

We claim that there is a basic open set $V$ in $\mathbf{P}_{L}^{n}$ such that $V \times_{L} M \subset U_{M}$, and thus $V \times_{L} K \subset U$. Namely, let $F_{1}, \ldots, F_{m} \in M\left[X_{0}, \ldots, X_{n}\right]$ be the images of $F$ under the elements of the Galois group $\operatorname{Gal}(M / L)$, and let $E=\prod F_{i}$. Then $E \in L^{\prime}\left[X_{0}, \ldots, X_{n}\right]$, where $L^{\prime}$ is the fixed field in $M$ of $\operatorname{Gal}(M / L)$. If $L$ has characteristic zero, then $L^{\prime}=L$ and so we may take $V$ to be the complement in $\mathbf{P}_{L}^{n}$ of the zero locus of $E$. If $L$ has characteristic $p \neq 0$, then $L^{\prime}$ is purely inseparable over $L$, say of degree $q=p^{r}$. Thus $E^{q} \in L\left[X_{0}, \ldots, X_{n}\right]$ and we may take $V$ to be the complement in $\mathbf{P}_{L}^{n}$ of the zero locus of $E^{q}$.

Now $V$ is nonempty, since it is a basic open set in $\mathbf{P}_{L}^{n}$. Since $L$ is infinite, $V$ contains $L$-valued points (by an easy induction on $n$ ). Choose such a point $P$, corresponding to some divisor in the linear system $\Delta$ over $L$. But this divisor induces a divisor $D$ over $K$ which is a member of the linear system $\Lambda$ over $K$ and which corresponds to the image of $P$ in the open set $U \subset \mathbf{P}_{K}^{n}$. So indeed the divisor $D$, which is defined over $L$, has the property in question. Q.E.D.

\section{COMPLETE INTERSECTIONS}

For this section we fix a projective nonsingular variety $X$ of dimension $n$ and a very ample divisor $D$ on $X$. By a hypersurface we mean a divisor from the linear system $|m D|$ for some $m \geq 1$. A purely dimension $l$ closed subscheme $Y$ of $X$ will be called a complete intersection if there exist $n-l$ hypersurfaces $H_{1}, \ldots, H_{n-l}$ such that $Y=H_{1} \cap \cdots \cap H_{n-l}$ scheme theoretically; that is, on any affine patch of $X$ the ideal of $Y$ is the sum of the ideals of the $H_{i}$ 's. The following theorem was proved by Nishi [N] in the special case that $X=\mathbf{P}^{n}$ and $D$ is a hyperplane.

(3.1) Theorem. Let $Y$ be a nonsingular closed subscheme of $X$ of dimension l. Assume that $2 l<n$. Then there exist distinct nonsingular hypersurfaces $H_{1}, \ldots, H_{n-2 l}$ containing $Y$ such that for each $i, 1 \leq i \leq n-2 l, H_{1} \cap \cdots \cap H_{i}$ is a nonsingular complete intersection of dimension $n-i$.

Proof. If we think of $X$ as embedded in projective space $\mathbf{P}^{N}$, then we know that the homogeneous ideal of $Y$ in $\mathbf{P}^{N}$ will be finitely generated. This says that for $k \gg 0$ the subsystem of $|k D|$ of all divisors containing $Y$ will have $Y$ as its scheme theoretic base locus. If we let $\pi: X^{\prime} \rightarrow X$ be the blow-up of $X$ along $Y$ with exceptional divisor $E$, then again, for $k \gg 0, \pi^{*}(k D)-E$ is very ample on $X^{\prime}$ [H1, exercise II.5.12, Proposition II.7.10]. Since $2 l<n$, Theorem 2.1 then applies to tell us that a general divisor of $|k D|$ containing $Y$ will be a nonsingular hypersurface in $X$ containing $Y$. Pick one such hypersurface and call it $H_{1}$.

We now proceed inductively. Having chosen $H_{1}, \ldots, H_{j}$, one chooses $H_{j+1}$ 
as follows. By the previous paragraph there is clearly no problem in finding a nonsingular hypersurface $H_{j+1}$ containing $Y$; the problem is making sure that $H_{1} \cap \cdots \cap H_{j+1}$ is also nonsingular. To do this, apply the previous argument used to obtain $H_{1}$ to the linear system given by the restriction of $k^{\prime} D$ (for appropriate $k^{\prime} \geq k$ ) to $H_{1} \cap \cdots \cap H_{j}$. Q.E.D.

(3.2) Corollary. If $\operatorname{dim} Y<\frac{1}{2} \operatorname{dim} X$, then $Y$ is contained in a nonsingular complete intersection subvariety of dimension equal to $2 \operatorname{dim} Y$.

For example, a nonsingular curve in $\mathbf{P}^{n}$ lies on a nonsingular complete intersection surface. See also [N].

Remarks. (a) It is easy to see that a complete intersection of dimension at least one is connected. Namely, a general complete intersection-i.e., the intersection of general hypersurfaces-is connected because the intersections can be viewed successively as very ample divisors on nonsingular varieties. Any complete intersection is a flat specialization of a general complete intersection and hence still connected.

(b) Nishi [N] gives an example to show that the numerical restriction $2 l<n$ is needed. We consider this example from a more abstract point of view, using a suggestion of Donu Arapura. According to the Grothendieck-Lefshetz theorem [H2, p. 179], if $Y$ is a subscheme of dimension $\geq 3$ that is a (strict) complete intersection in $\mathrm{P}_{K}^{n}$, then Pic $Y \cong Z$ and is generated by the class $\mathscr{O}_{Y}(1)$. It follows that a subvariety of dimension at least 2 in projective space that is not a complete intersection cannot be a Cartier divisor on a complete intersection. For $\mathbf{P}^{4}$ this says that a nonsingular surface that is not a complete intersection cannot be contained in a nonsingular hypersurface. (There are nonsingular surfaces in $\mathbf{P}^{4}$ that are not complete intersections. For instance, embed the blow-up of $\mathbf{P}^{2}$ at a point into $\mathbf{P}^{4}$ by the system of conics through that point. The degree of the image is three, so it cannot be a complete intersection.)

(3.3) Corollary. In (3.1) allow $Y$ to have isolated singularities but require that all these singularities have tangent spaces of dimensions $\leq k$. Set $i=$ $\min (n-2 l, n-k)$. Then there exist distinct nonsingular hypersurfaces $H_{1}, \ldots$, $H_{i}$ containing $Y$ such that for each $j, 1 \leq j \leq i, H_{1} \cap \cdots \cap H_{j}$ is a nonsingular complete intersection of dimension $n-j$.

Proof. Follow the proof of (3.1), using (2.7) where needed. Q.E.D.

In particular, a curve in $\mathbf{P}^{n}$ with isolated planar singularities is contained in a nonsingular surface.

(3.4) Theorem. Let $Y$ be a nonsingular closed subscheme of $X$ with $Y$ of dimension $l$.

(i) If $2 l<n$, then there are infinitely many positive integers $d_{i}$ such that there is a nonsingular hypersurface $H_{i} \in\left|d_{i} D\right|$ containing $Y$. 
(ii) If $2 l \geq n$, then there are at most $n-l$ positive integers $d_{i}$ such that there is a nonsingular hypersurface $H_{i} \in\left|d_{i} D\right|$ containing $Y$. If $Y=H_{1} \cap \cdots \cap H_{n-l}$ is a complete intersection, then any nonsingular hypersurface containing $Y$ must be linearly equivalent to one of these $H_{i}$.

Proof. Statement (i) follows from the proof of (3.1). To prove statement (ii) let $H$ be a nonsingular hypersurface containing $Y$. If $C$ denotes the conormal bundle to $Y$ in $X$, then $H$ gives a nowhere vanishing section of $C \otimes \mathscr{O}(H)$ on $Y$ (see (2.3) and surrounding discussion). This means that $(n-l)$ th Chern class of $C \otimes \mathscr{O}(H)$ must be zero. From standard Chern class calculations (see $[\mathrm{F}])$ we see that

$$
c_{n-l}(C \otimes \mathscr{O}(H))=\sum_{i=0}^{n-l} c_{i}(C) H^{n-l-i} .
$$

Say $H \in|m D|$ and the degree of $c_{i}(C) \cdot D^{l-i}$ is $k_{i}$. If $c_{n-l}(C \otimes \mathscr{O}(H))=0$ then

$$
\sum_{i=0}^{n-l} k_{i} m^{n-l-i}=0 .
$$

This is a polynomial of degree $n-l$ in $m$, so it has at most $n-l$ solutions.

Now suppose $Y$ is a complete intersection $Y=H_{1} \cap \cdots \cap H_{n-l}$ with $H_{j} \in$ $\left|m_{j} D\right|$. For any containment $Z \subset W$ of nonsingular schemes we have the following exact sequence involving tangent and normal bundles.

$$
0 \rightarrow T_{Z} \rightarrow T_{W \mid Z} \rightarrow N_{Z} W \rightarrow 0 \text {. }
$$

Some of the $H_{i}$ may be singular, but they all must be nonsingular along $Y$. We also know that the normal bundle to a Cartier divisor $E$ is $\mathscr{O}_{E}(E)$. Using these facts, one explicitly computes the Chern classes of $C$ and the $k_{i}$ in (3.5) (up to knowing $D^{l}$ ) and finds that the roots of (3.5) are exactly the $m_{j}$. Q.E.D.

(3.6) Remark. Consider equation (3.5). One would expect that a random polynomial would not have any positive integer zeros. Furthermore, the vanishing of the polynomial in (3.5) only says that $c_{n-l}(C \otimes \mathscr{O}(m D))$ has degree zero with respect to $D$, not that it actually is zero. If the Chern classes of $C$ involve terms other than powers of $D$ (as they might if $Y$ is not a complete intersection), then it is even more unlikely that $c_{n-l}(C \otimes \mathscr{O}(m D))$ will ever be zero. Finally, we have only shown that the vanishing of $c_{n-l}(C \otimes \mathscr{O}(m D))$ is a necessary condition for $Y$ to be contained in a nonsingular hypersurface. All this leads one to suspect that it is very difficult for a subvariety of $X$ of dimension $\geq \frac{1}{2} n$ to be contained in a nonsingular hypersurface. But it is unclear how to make this observation into a precise assertion.

Remark. As at the end of $\S 2$, suppose that $L \subset K$ is an infinite subfield. Assume also that $X$ and the linear system $|D|$ are defined over $L$. Then, as at the end of $\S 2$, one may show that the hypersurfaces occurring in (3.1), (3.3), and (3.4)(i) (for most $d_{i}$ ) can be chosen to be defined over $L$. 


\section{LIAISON}

Continue with the assumptions of $\S 3$ but assume further that $X=\mathbf{P}^{n}$ and $D$ is a hyperplane. Given two reduced closed subschemes $Y_{1}$ and $Y_{2}$ of $\mathbf{P}^{n}$, we say that $Y_{1}$ and $Y_{2}$ are directly linked in liaison if the scheme-theoretic union $Y_{1} \cup Y_{2}$ (locally defined by the intersection of the ideals of $Y_{1}$ and $Y_{2}$ ) is a complete intersection, and no irreducible component of either of the subschemes is contained in the other. Thus $Y_{2}$ is minimal among subschemes $Y$ of $\mathbf{P}^{n}$ such that $Y_{1} \cup Y=Y_{1} \cup Y_{2}$. Consider the homogeneous coordinate ring $S$ of $\mathbf{P}^{n}$ :

$$
S=\bigoplus_{i=0}^{\infty} H^{0}\left(\mathbf{P}^{n}, \mathscr{O}(i)\right)=K\left[X_{0}, \ldots, X_{n}\right]
$$

A subscheme $Y$ of $\mathbf{P}^{n}$ of pure dimension $l$ can be represented as a complete intersection $Y=H_{1} \cap \cdots \cap H_{n-l}$ if and only if the $H_{i}$ generate the homogeneous ideal of $Y$ in $S$; see [H1, exercise II.8.4(a)].

(4.1) Theorem. Let $Y \subset \mathbf{P}^{n}$ be a nonsingular closed subscheme of pure dimension $l$ that is a complete intersection. If $l \geq \frac{2}{3} n$, then $Y$ cannot be directly linked to any nonsingular closed subscheme of $\mathbf{P}^{n}$ that is not a complete intersection.

Proof. Say $Y$ is directly linked to a nonsingular $Y^{\prime}$. Thus $Y=H_{1} \cap \cdots \cap H_{n-l}$ and $Y \cup Y^{\prime}=H_{1}^{\prime} \cap \cdots \cap H_{n-l}^{\prime}$. We may assume that in the expressions for $Y$ and $Y \cup Y^{\prime}$, the $H$ 's are listed so that their degrees are nondecreasing. We wish to show that $Y^{\prime}$ is a complete intersection.

(4.2) Claim. For some $m, 1 \leq m \leq n-l$, we may choose $H_{i}$ and $H_{i}^{\prime}$ above so that

(i) $H_{i}^{\prime}=H_{i}$ for $m<i \leq n-l$,

(ii) $\left(H_{1}^{\prime}, \ldots, H_{m}^{\prime}\right) \subset\left(H_{1}, \ldots, H_{m}\right)$,

(iii) $\operatorname{deg} H_{m}^{\prime}>\operatorname{deg} H_{m}$.

Proof of (4.2). Note that (i) holds trivially with $m=n-l$; let $j \geq 0$ be the least value of $m$ for which there is a choice of $H_{i}, H_{i}^{\prime}$ satisfying (i). Here $j \geq 1$, since $Y \neq Y \cup Y^{\prime}$. Observe that for some such choice of $H_{i}, H_{i}^{\prime}$, property (ii) also holds. Namely, first make any choice satisfying (i) as above; thus the inclusion $Y \subset Y \cup Y^{\prime}$ implies that we may write

$$
H_{i}^{\prime}=\sum_{k=1}^{n-l} G_{i k} H_{k}=\sum_{k=1}^{j} G_{i k} H_{k}+\sum_{k=j+1}^{n-l} G_{i k} H_{k}^{\prime} \quad \text { for } 1 \leq i \leq j,
$$

where the $G_{i k}$ are homogeneous polynomials, with $\operatorname{deg} G_{i k}+\operatorname{deg} H_{k}=\operatorname{deg} H_{i}^{\prime}$ for all $i$ and $k$. Replacing $H_{i}^{\prime}$ by $\sum_{k=1}^{j} G_{i k} H_{k}$ for $1 \leq i \leq j$, and leaving the other $H_{i}^{\prime}$ and all of the $H_{i}$ as before, we obtain a choice satisfying (i) and (ii).

It remains to show that (possibly after again altering our choice) we may satisfy (iii) as well. With $G_{i k}$ as above, note that $G_{h j} \neq 0$ for some $h \leq j$; 
otherwise, we would have the containment $\left(H_{1}^{\prime}, \ldots, H_{j}^{\prime}\right) \subset\left(H_{1}, \ldots, H_{j-1}\right)$, which is impossible because the zero set of the former ideal has smaller dimension than that of the latter. So with $h$ as above, $\operatorname{deg} H_{j}^{\prime} \geq \operatorname{deg} H_{h}^{\prime}=$ $\operatorname{deg} G_{h j}+\operatorname{deg} H_{j} \geq \operatorname{deg} H_{j}$. If the inequality is strict, we are done. Otherwise, we have that $\operatorname{deg} G_{h j}=0$; i.e., $G_{h j}$ is a nonzero constant. Also, since the $H_{k}^{\prime}$ are listed in order of nondecreasing degree, we have $\operatorname{deg} H_{j}^{\prime}=\operatorname{deg} H_{h}^{\prime}$. So interchanging $H_{h}^{\prime}$ and $H_{j}^{\prime}$, we may assume that $G_{j j} \neq 0$. Multiplying $H_{j}^{\prime}$ by the constant $G_{j j}$, we have that $H_{j}^{\prime}=H_{j}+$ terms involving lower $H_{i}$. Thus we may replace $H_{j}$ by $H_{j}^{\prime}$. So (i) holds with $m=j-1$, contradicting the minimality of $j$. Therefore (iii) does in fact hold for $m=j$ and the above choice of $H_{i}$ and $H_{i}^{\prime}$, and the claim is shown.

(4.3) Claim. Let $m$ be as in (4.2), let $1<k \leq m$, and suppose that some choice of $H_{i}, H_{i}^{\prime}$ satisfies the condition

$\left(C_{k}\right) \quad Y \cup Y^{\prime}=H_{1}^{\prime} \cap \cdots \cap H_{k-1}^{\prime} \cap H_{k+1} \cap \cdots \cap H_{n-l} \cap H_{m}^{\prime}$.

Then

(1) $\operatorname{deg} H_{k-1}^{\prime} \leq \operatorname{deg} H_{k}$ and

(2) the singular locus of $H_{m}^{\prime}$ is nonempty and meets $Y$ in codimension at most $m$ on $Y$.

Proof of (4.3). Make some choice of $H_{i}, H_{i}^{\prime}$ as in (4.2). Using $\left(C_{k}\right)$ and the inclusion $Y \subset Y \cup Y^{\prime}$, and proceeding as in the first paragraph of the proof of (4.2), we may alter our choice of $H_{1}^{\prime}, \ldots, H_{k-1}^{\prime}$ (without changing their degrees, and while leaving alone the other $H_{i}^{\prime}$ and all of the $\left.H_{i}\right)$ so that the ideal they generate is contained in the ideal $\left(H_{1}, \ldots, H_{k}\right)$.

Now suppose that $h, j \leq m$ and that we have the containment of ideals $\left(H_{1}^{\prime}, \ldots, H_{j}^{\prime}\right) \subset\left(H_{1}, \ldots, H_{h}\right)$. Then the locus of $H_{j}^{\prime}$ contains the complete intersection $H_{1} \cap \cdots \cap H_{h}$. Now $H_{1} \cap \cdots \cap H_{h}$ may be singular, but since it is a complete intersection of Cartier divisors it still has a conormal bundle $C$ whose Chern classes may be computed as in the proof of (3.4). If, in addition, the degree of $H_{j}^{\prime}$ is greater than the degrees of $H_{1}, \ldots, H_{h}$, then the Chern class $c_{n-h}\left(C \otimes \mathscr{O}\left(H_{j}^{\prime}\right)\right)$ is nonzero. Restricting this class to $Y$, we see that the singularities of $H_{j}^{\prime}$ on $Y$ occur in codimension at most $h$ in $Y$.

In particular, by (4.2) we may take $h=j=m$ and thus obtain (2). Meanwhile, if (1) fails, then by the first paragraph of this proof we may take $j=k-1$ and $h=k$ and conclude that $H_{k-1}^{\prime}$ has singularities of codimension at most $k$ in $Y$. Furthermore, the singular locus supports a class of codimension $k$ in $Y$ which is a multiple of a power of the restriction of $D \sim \mathscr{O}(1)$ to $Y$, and therefore meets everything on $Y$ that it dimensionally should. Since $l \geq \frac{2}{3} n$, we have $m+k \leq 2 m \leq 2(n-l) \leq l$, and so the singularities of $H_{k-1}^{\prime}$ meet the singularities of $H_{m}^{\prime}$ on $Y$. At any such point of intersection, the multiplicities of $H_{k-1}^{\prime}$ and of $H_{m}^{\prime}$ are each at least 2, and so the multiplicity of 
$Y \cup Y^{\prime}=H_{1}^{\prime} \cap \cdots \cap H_{n-l}^{\prime}$ must be at least 4. But this cannot happen since both $Y$ and $Y^{\prime}$ are nonsingular. This contradiction implies the claim.

(4.4) Claim. With $m$ as in (4.2), there is a $k(1 \leq k \leq m)$ such that some choice of $H_{i}$ and $H_{i}^{\prime}$ will satisfy the condition

$\left(D_{k}\right) \quad Y \cup Y^{\prime}=H_{1} \cap \cdots \cap H_{k-1} \cap H_{k+1} \cap \cdots \cap H_{n-l} \cap H_{m}^{\prime}$.

Proof of (4.4). By (4.2)(ii), the locus of that $H_{m}^{\prime}$ contains $H_{1} \cap \cdots \cap H_{m}$, which is a complete intersection. For each $k(1 \leq k \leq m)$, we may consider the condition $\left(C_{k}\right)$ of (4.3). Thus $\left(C_{1}\right)=\left(D_{1}\right)$, and $\left(C_{m}\right)$ is immediate from (4.2). It thus suffices to show that for $1<k \leq m$, if there is a choice of $H_{i}$ and $H_{i}^{\prime}$ satisfying $\left(C_{k}\right)$, then there also exists a choice which satisfies $\left(D_{k}\right)$ or $\left(C_{k-1}\right)$.

So suppose $\left(C_{k}\right)$ holds for some $H_{i}, H_{i}^{\prime}$. As shown in the proof of (4.3), we may choose the $H_{i}$ and $H_{i}^{\prime}$ such that $\left(H_{1}^{\prime}, \ldots, H_{k-1}^{\prime}\right) \subset\left(H_{1}, \ldots, H_{k}\right)$. There are two possibilities:

Case (a). $\left(H_{1}^{\prime}, \ldots, H_{k-1}^{\prime}\right) \subset\left(H_{1}, \ldots, H_{k-1}\right)$.

It suffices to show that the inclusion must be an equality. For if this is shown, then we may replace $H_{i}^{\prime}$ by $H_{i}$ for $1 \leq i \leq k-1$, and then $\left(D_{k}\right)$ holds.

So suppose that inclusion is strict. Since both of these ideals must have reduced complete intersection zero sets of the same dimension, we conclude that the zero set of $\left(H_{1}^{\prime}, \ldots, H_{k-1}^{\prime}\right)$ is reducible. Thus $H_{1}^{\prime} \cap \cdots \cap H_{k-1}^{\prime}$ has two components, and they meet in codimension one ([H3] and the remark following (3.2)). Now $H_{1} \cap \cdots \cap H_{k-1}$ must be nonsingular along $Y$, since $Y$ is nonsingular and $Y=\left(H_{1} \cap \cdots \cap H_{k-1}\right) \cap\left(H_{k} \cap \cdots \cap H_{n-l}\right)$. Also, since $Y$ is a complete intersection, it must meet every cycle on $H_{1} \cap \cdots \cap H_{k-1}$ that it dimensionally should meet. Thus $H_{1}^{\prime} \cap \cdots \cap H_{k-1}^{\prime}$ is singular in codimension at most one on $Y$, namely along the locus in $Y$ of the intersection of the two components. These singularities then meet the singularities of $H_{m}^{\prime}$ on $Y$ (see (4.3)(2)). As in the proof of (4.3), this contradicts the nonsingularity of $Y$ and $Y^{\prime}$, thus proving the claim in this case.

Case (b). $\left(H_{1}^{\prime}, \ldots, H_{k-1}^{\prime}\right)$ is not contained in $\left(H_{1}, \ldots, H_{k-1}\right)$.

By (4.3), we have $\operatorname{deg} H_{k-1}^{\prime} \leq \operatorname{deg} H_{k}$. So, as in the proof of (4.2), we have (possibly after renumbering and multiplying by a nonzero constant) that $H_{k-1}^{\prime}=H_{k}+$ terms involving lower $H_{i}$. Thus we may replace $H_{k}$ by $H_{k-1}^{\prime}$ and still have the same ideal. Thus $\left(C_{k-1}\right)$ holds.

(4.5) Completion of the proof of (4.1). Let $m$ and $k$ be as in (4.4). For the corresponding choice of $H_{i}$ and $H_{i}^{\prime}$, the condition $\left(D_{k}\right)$ and the inclusion $Y \subset$ $Y \cup Y^{\prime}$ imply that we may write $H_{m}^{\prime}=\sum_{j=1}^{n-l} G_{j} H_{j}$, where $G_{j}$ is homogeneous and $\operatorname{deg} G_{j}+\operatorname{deg} H_{j}=\operatorname{deg} H_{m}^{\prime}$. Thus

$$
\begin{aligned}
Y \cup Y^{\prime} & =H_{1} \cap \cdots \cap H_{k-1} \cap H_{k+1} \cap \cdots \cap H_{n-l} \cap H_{m}^{\prime} \\
& =H_{1} \cap \cdots \cap H_{k-1} \cap H_{k+1} \cap \cdots \cap H_{n-l} \cap G_{k} H_{k},
\end{aligned}
$$


scheme-theoretically. Since $Y \cup Y^{\prime}$ is reduced, $G_{k}$ and $H_{k}$ have no common factors, and so the locus of $G_{k} H_{k}$ is the scheme-theoretic union of that of $G_{k}$ and that of $H_{k}$. So

$$
Y \cup Y^{\prime}=H_{1} \cap \cdots \cap H_{k-1} \cap H_{k+1} \cap \cdots \cap H_{n-l} \cap\left(G_{k} \cup H_{k}\right)=Y \cup Y^{\prime \prime},
$$

where $Y^{\prime \prime}=H_{1} \cap \cdots \cap H_{k-1} \cap H_{k+1} \cap \cdots \cap H_{n-l} \cap G_{k}$, which is a complete intersection. Now by the definition of liaison, $Y^{\prime}$ is minimal among subschemes of $\mathrm{P}^{n}$ whose union with $Y$ is $Y \cup Y^{\prime}$. So $Y^{\prime} \subset Y^{\prime \prime}$. But

$$
\begin{aligned}
\operatorname{deg} Y+\operatorname{deg} Y^{\prime} & =\operatorname{deg} Y \cup Y^{\prime}=\left[\prod_{i \neq k} h_{i}\right]\left[g_{k}+h_{k}\right] \\
& =\prod_{i=1}^{n-l} h_{i}+\left[\prod_{i \neq k} h_{i}\right] g_{k}=\operatorname{deg} Y+\operatorname{deg} Y^{\prime \prime},
\end{aligned}
$$

where $h_{i}=\operatorname{deg} H_{i}$ and $g_{k}=\operatorname{deg} G_{k}$. So in fact $Y^{\prime}=Y^{\prime \prime}$. Thus $Y^{\prime}$ is a complete intersection. Q.E.D.

One might wonder whether the $\frac{2}{3}$ in (4.1) is in any way related to the $\frac{2}{3}$ in the following conjecture of Hartshorne [H4].

Conjecture (Hartshorne). If $Y$ is a nonsingular subvariety of dimension $r$ of $\mathbf{P}^{n}$ and $r>\frac{2}{3} n$, then $Y$ is a complete intersection.

Notice that if Hartshorne's conjecture is true, then Theorem (4.1) is automatic except possibly when $l=\frac{2}{3} n$. Hartshorne has shown by example that the strict inequality in this conjecture cannot be replaced by a $\geq$. Namely, the Grassmannian $G$ of lines in $\mathbf{P}^{4}$ embeds via the Plucker embedding as a 6 -fold in $\mathbf{P}^{9}$ that is not a complete intersection. Note that this provides a nontrivial example of Theorem (4.1), namely that $G$ with this embedding cannot be directly linked to a nonsingular complete intersection. Also observe that in the absence of a proof of Hartshorne's conjecture, Theorem (4.1) does indicate one place not to look for a counterexample to Hartshorne's conjecture. Namely, it says that a counterexample (if one exists) could not be directly linked to a nonsingular complete intersection. See also [HU], where Theorem 4.1 is asserted in the strict inequality case, via other methods.

\section{REFERENCES}

[F] William Fulton, Intersection theory, Springer-Verlag, Berlin, 1984.

[H1] Robin Hartshorne, Algebraic geometry, Springer-Verlag, New York, 1977.

[H2] Ample subvarieties of projective varieties, Lecture Notes in Math., vol. 156, SpringerVerlag, Heidelberg, 1970.

[H3] _- Complete intersections and connectedness, Amer. J. Math. 84 (1962), 497-508.

[H4] _ Varieties of small codimension in projective space, Bull. Amer. Math. Soc. 80 (1974), 1017-1032.

[HU] Craig Huneke and Bernd Ulrich, The structure of linkage, Ann. of Math. 126 (1987), 277-334. 
[N] Mieo Nishi, On the imbedding of a nonsingular variety in an irreducible complete intersection, Mem. Coll. Sci. Univ. Kyoto Ser. A Math. 29 (1955), 172-187.

[Z] Oscar Zariski, The theorem of Bertini on variable singular points of a linear system of varieties, Trans. Amer. Math. Soc. 56 (1944), 130-140.

Department of Mathematics, University of Pennsylvania, Philadelphia, PennsylvaNIA 19104-6395 (Current address of David Harbater)

Current address (Steven Diaz): Department of Mathematics, Syracuse University, Syracuse, New York 13244-1150 